\title{
Longitudinally Mapping Childhood Socioeconomic Status Associations with Cortical and Subcortical Morphology
}

\author{
CCassidy L. McDermott, ${ }^{1}$ Jakob Seidlitz, ${ }^{1}$ CAjay Nadig, ${ }^{1}$ Siyuan Liu, ${ }^{1}$ Liv S. Clasen, ${ }^{1}$ Jonathan D. Blumenthal, ${ }^{1}$ \\ Paul Kirkpatrick Reardon, ${ }^{1}$ François Lalonde, ${ }^{1}$ Deanna Greenstein, ${ }^{2}$ - Raihaan Patel, ${ }^{3}$ M. Mallar Chakravarty, ${ }^{3,4}$ \\ Jason P. Lerch, ${ }^{5}$ and ${ }^{\circ}$ Armin Raznahan ${ }^{1}$ \\ ${ }^{1}$ Developmental Neurogenomics Unit, Human Genetics Branch, National Institute of Mental Health, Bethesda, Maryland 20892, ${ }^{2}$ Experimental \\ Therapeutics and Pathophysiology Branch, National Institute of Mental Health, Bethesda, Maryland 20892, ${ }^{3}$ Cerebral Imaging Centre, Douglas Mental \\ Health University Institute, McGill University, Montreal, Quebec, Canada H4H 1R3, ${ }^{4}$ Department of Psychiatry, McGill University, Montreal, Quebec, \\ Canada H3A OG4, and 5Department of Medical Biophysics, University of Toronto, Toronto, Ontario, Canada M5G 1L7
}

Childhood socioeconomic status (SES) impacts cognitive development and mental health, but its association with human structural brain development is not yet well characterized. Here, we analyzed 1243 longitudinally acquired structural MRI scans from 623 youth (299 female/324 male) to investigate the relation between SES and cortical and subcortical morphology between ages 5 and 25 years. We found positive associations between SES and total volumes of the brain, cortical sheet, and four separate subcortical structures. These associations were stable between ages 5 and 25. Surface-based shape analysis revealed that higher SES is associated with areal expansion of lateral prefrontal, anterior cingulate, lateral temporal, and superior parietal cortices and ventrolateral thalamic, and medial amygdalohippocampal subregions. Meta-analyses of functional imaging data indicate that cortical correlates of SES are centered on brain systems subserving sensorimotor functions, language, memory, and emotional processing. We further show that anatomical variation within a subset of these cortical regions partially mediates the positive association between SES and IQ. Finally, we identify neuroanatomical correlates of SES that exist above and beyond accompanying variation in IQ. Although SES is clearly a complex construct that likely relates to development through diverse, nondeterministic processes, our findings elucidate potential neuroanatomical mediators of the association between SES and cognitive outcomes.

Key words: brain development; cortical surface area; longitudinal; socioeconomic status; structural MRI

Significance Statement

Childhood socioeconomic status (SES) has been associated with developmental disparities in mental health, cognitive ability, and academic achievement, but efforts to understand underlying SES- brain relationships are ongoing. Here, we leverage a unique developmental neuroimaging dataset to longitudinally map the associations between SES and regional brain anatomy at high spatiotemporal resolution. We find widespread associations between SES and global cortical and subcortical volumes and surface area and localize these correlations to a distributed set of cortical, thalamic, and amygdalo-hippocampal subregions. Anatomical variation within a subset of these regions partially mediates the positive relationship between SES and IQ. Our findings help to localize cortical and subcortical systems that represent candidate biological substrates for the known relationships between SES and cognition.

\section{Introduction}

Early brain development occurs within the context of each child's experiences and environment, which vary significantly as a func-

\footnotetext{
Received July 13, 2018; revised 0ct. 31, 2018; accepted Nov. 16, 2018.

Author contributions: C.L.M. wrote the first draft of the paper; C.L.M., J.S., A.N., and A.R. edited the paper; C.L.M., J.S., and A.R. designed research; L.S.C., J.D.B., and F.L. performed research; R.P., M.M.C., and J.P.L. contributed unpublished reagents/analytic tools; C.L.M., S.L., P.K.R., D.G., and A.R. analyzed data; C.L.M. and A.R. wrote the paper.

This work was supported by the Intramural Research Program of the National Institutes of Mental HealthNational Institutes of Health (1ZIAMH002949-01 under protocol NCT00001246). We thank the participants and families who took part in this study.

The authors declare no competing financial interests.

Correspondence should be addressed to Armin Raznahan at raznahana@mail.nih.gov.
}

tion of socioeconomic status (SES). Childhood SES is typically measured by factors including parental income, education, and occupation (Hollingshead, 1957; McLoyd, 1998) and has been associated with disparate outcomes in mental health, cognitive development, and academic achievement (Brooks-Gunn and Duncan, 1997; Sirin, 2005; Noble et al., 2007; Reiss, 2013). These associations are thought to arise through diverse causal pathways, including: (1) direct SES-linked effects on cognitive and health outcomes (Ritsher et al., 2001; Kendler et al., 2015), (2) the ca-

https://doi.org/10.1523/JNEUROSCl.1808-18.2018

Copyright $\odot 2019$ the authors $\quad 0270-6474 / 19 / 391365-09 \$ 15.00 / 0$ 
pacity of mental health or cognitive challenges to negatively impact SES (Tiikkaja et al., 2016), and (3) factors that simultaneously increase risk for lowered SES and cognitive difficulties ( $\mathrm{Tr}$ zaskowski et al., 2014; Hill et al., 2016).

The robust epidemiological data connecting childhood SES to behavioral and cognitive development have motivated recent neuroimaging studies aimed at mapping SES effects on brain anatomy. SES has been positively associated with total gray matter volume and less consistently with white matter volume (Luby et al., 2013; Hair et al., 2015; Gianaros et al., 2017), as well as with volume in regions of a priori interest, including the prefrontal cortex and hippocampus (Jednoróg et al., 2012; Noble et al., 2012; Hanson et al., 2013; Luby et al., 2013; Hair et al., 2015; Holz et al., 2015). Furthermore, a recent landmark study (Noble et al., 2015) mapped crosssectional associations between socioeconomic factors and cortical surface area. These studies suggest that observed associations between childhood SES and neurodevelopmental outcomes may be centered on specific brain systems and raise as yet unresolved questions regarding when SES-neuroanatomy associations are established, whether they are predominantly cortical or subcortical, and how correlations between SES and neuroanatomy relate to accompanying variation in cognitive ability (Noble et al., 2007; Brito and Noble, 2014).

Here, we sought to build on the current understanding of SES and brain development in five key directions. First, to determine whether SES associations with neuroanatomy are developmentally dynamic or stable in this age range (Giedd et al., 1999), we model SES-brain associations in a longitudinal sample of 1243 structural neuroimaging scans from 623 healthy individuals ages $5-25$. We combine two complementary approaches to analysis of longitudinal data: mixed models (Pinheiro et al., 2016) and direct analysis of intra-individual estimates of anatomical change computed from repeat scans. Second, we separately model SES associations with regional cortical thickness (CT) and surface area (SA), two biologically distinct phenotypes that together determine cortical volume (Raznahan et al., 2011). Findings remain sparse and mixed regarding the relative strength and spatial distribution of SES relations with SA and CT (Lawson et al., 2013; Noble et al., 2015). Third, we extend our analyses to assess the relation between SES and the anatomy of five major noncortical structures (henceforth "subcortical" structures) using multi-atlas methods that provide both bulk volume estimates and spatially fine-grained measures of shape. Simultaneous examination of SES associations with cortical and subcortical anatomy is critical given evidence that these brain systems function (Redgrave et al., 2010), develop (Raznahan et al., 2014) and connect (Draganski et al., 2008) in a topographically organized manner. Fourth, we formally characterize functional associations of those cortical regions that correlate with SES using the Neurosynth platform for meta-analysis of neuroimaging data (Yarkoni et al., 2011). Finally, given previously noted positive associations between SES and cognitive performance and the potential of brain anatomy to vary as a function of cognitive ability (Walhovd et al., 2016), we probe the complex associations between SES, neuroanatomy, and cognition through two methods that have been used separately in previous literature: (1) including IQ as a covariate in models of SES effects on neuroanatomy (Noble et al., 2012; Lawson et al., 2013) and (2) assessing whether structural phenotypes correlated with SES mediate the relation between SES and IQ (Hair et al., 2015; Noble et al., 2015).
Table 1. Participant characteristics

\begin{tabular}{ll}
\hline Characteristic & Data \\
\hline No. of individuals & 623 \\
No. of scans & 1243 \\
Sex, $n$ & \\
$\quad$ Female & 299 \\
$\quad$ Male & 324 \\
Age at first scan, years & \\
$\quad$ Mean (SD) & $12.0(4.0)$ \\
$\quad$ Range & $5.2-25.4$ \\
Handedness, $n$ & \\
$\quad$ Left & 35 \\
Right & 537 \\
$\quad$ Mixed & 51 \\
Race, $n$ & \\
$\quad$ White & 534 \\
Black & 40 \\
Asian & 14 \\
Hispanic & 20 \\
Other & 15 \\
IQ & \\
Mean (SD) & $114(12.4)$ \\
Range & $78-150$ \\
Hollingshead SES & \\
Mean (SD) & $41.2(18.3)$ \\
Range & $95-20$ \\
No. of scans per individual & \\
1 scan & 279 \\
2 scans & 168 \\
$\geq 3$ scans & 176 \\
\hline & \\
\hline & \\
\hline
\end{tabular}

\section{Materials and Methods}

Participants. This study includes a longitudinal sample of 1243 structural magnetic resonance imaging (sMRI) brain scans from 623 healthy children and adolescents between 5 and 25 years old (Table 1). Participants were recruited through local advertisement for a study of typical brain development conducted at the National Institute of Mental Health Intramural Research Program between 1990 and 2010. Participants were screened and excluded on the basis of a history of mental health treatment, use of psychiatric medication, enrollment in special services at school, or diagnosis of any medical condition known to affect the nervous system. The research protocol was approved by the institutional review board at the National Institute of Mental Health and written informed consent or assent was obtained from all children who participated in the study, as well as consent from their parents if the child was under the age of 18 .

Socioeconomic status. Childhood socioeconomic status (SES) was quantified using the Amherst modification of the Hollingshead twofactor index (Hollingshead, 1957; Watt, 1976). Parental education and occupation were each coded on a seven-point scale and these two values were used to derive and record a single SES score that was used for analyses. When education and occupation were reported for two parents, the highest SES was used. In the resulting index, children from the most advantaged families receive the lowest Hollingshead score of 20, whereas children from the most disadvantaged families receive the highest Hollingshead score of 134 . For ease of interpretation, we refer to SES variation using conventional directionality such that "higher SES" refers to a lower Hollingshead score. Accordingly, reported positive associations with SES in the manuscript indicate variables that increase in value with greater SES and are thus negatively correlated with Hollingshead score. In graphical representation of such associations, $x$-axis scales for SES are reversed such that right-most values index the highest SES levels (i.e., lowest Hollingshead scores).

General cognitive ability. Full-scale IQ was estimated for each child in the sample using an age-appropriate Wechsler scale. The majority $(N=$ 562) of children received the Wechsler Abbreviated Scale of Intelligence (Wechsler, 1999) and other scales used include the WAIS-R, WISC-R, 
and WISC-III and the WPPSI and WPPSI-III. Of the 344 individuals with repeat assessments, only 60 had IQ measures within 1 year of at least 2 scans. We therefore used the most recent complete IQ measurement for each individual.

Image acquisition and processing. All sMRI scans were T1-weighted images collected on the same $1.5 \mathrm{~T}$ General Electric SIGNA scanner with contiguous $1.5 \mathrm{~mm}$ axial slices using a 3D spoiled-gradient recalled-echo sequence (echo time $=5 \mathrm{~ms}$; repetition time $=24 \mathrm{~ms}$; flip angle $=45^{\circ}$; acquisition matrix $=256 \times 192$; number of excitations $=1$; field of view $=24 \mathrm{~cm}$ ). All scans passed visual assessment for motion artifact before processing.

Native sMRI scans were submitted to the CIVET 1.1.10 pipeline for automated morphometric analysis (Ad-Dab'bagh et al., 2006). The CIVET pipeline uses a validated neural net approach to voxel classification to calculate gray matter and white matter volume estimates (Zijdenbos et al., 2002; Cocosco et al., 2003) after initial correction of images for radiofrequency intensity nonuniformities (Collins et al., 1994; Sled et al., 1998). Following tissue classification, each image was fitted with two deformable mesh models to identify the inner and outer surface of cortical gray matter, and these surfaces were used to calculate cortical thickness and surface area at 40,962 vertices on each cortical hemisphere as described previously (MacDonald et al., 2000; Raznahan et al., 2012).

Subcortical segmentation and surface extraction were completed automatically using the MAGeT Brain algorithm (Chakravarty et al., 2013; Raznahan et al., 2014). Scans were first registered to the ICBM 152 template and corrected for radiofrequency intensity nonuniformities (Collins et al., 1994; Sled et al., 1998). For the striatum, thalamus, and pallidum, the segmentation atlas was created using a 3D reconstruction of serial histological data warped to an MRI-based template (Chakravarty et al., 2006). The MAGeT pipeline then customized this atlas to 21 randomly selected subjects within the sample. All 1243 scans were then warped to this set of templates, providing a set of 21 candidate subcortical segmentations for each scan. For the hippocampus and amygdala, five reference atlases were generated from high-resolution and high-contrast T1- and T2-weighted images from three males and two females using a 3 T scanner with final supersampled isotropic voxel dimensions of $0.3 \mathrm{~mm}$ (Wood, 2011). The MAGeT pipeline again created automated segmentation atlases for 21 randomly selected subjects, resulting in 105 possible segmentations (five atlases $\times 21$ templates) for each of the 1243 scans in our dataset. Each scan was labeled using the 21 striatum, thalamus, and pallidum segmentations and the 105 hippocampus and amygdala segmentations and the final segmentation was decided upon using a labelvoting procedure such that the label occurring most frequently at each voxel was retained. These procedures provided estimates of total bilateral volume for the hippocampus, amygdala, thalamus, striatum, and pallidum in each scan. All scans used for analysis passed visual quality control of these final subcortical segmentations to exclude visible segmentation errors of the five subcortical structures under study.

Surface-based representations of all five subcortical structures were then estimated on their respective atlases using a marching cubes algorithm (Lerch et al., 2008). Next, the nonlinear portions of the 21 transformations mapping each subject to the 21 input templates were concatenated and averaged across the template library to limit noise and increase precision and accuracy. These surface-based representations were warped to fit each template and each surface was warped to match each subject. This procedure yields 21 possible surface representations per subject for the striatum, thalamus, and pallidum and 105 possible surface representations for the hippocampus and amygdala, which were merged by estimating the median coordinate representation at each location. Next, a third of the surface area of each triangle forming the surface representation was assigned to each vertex within the triangle. The surface area at each vertex is the sum of all such assignments from all connected triangles. Finally, surface area values were blurred with a surface-based diffusion-smoothing kernel ( $5 \mathrm{~mm}$ for the amygdala, hippocampus, striatum, and thalamus and $3 \mathrm{~mm}$ for the pallidum). This processing stream generated surface area values for a total of 26,401 vertices across the five subcortical structures in each scan.

Experimental design and statistical analyses. The effect of SES on each anatomical metric of interest was modeled using a linear mixed-effects model with sex and centered age as fixed effects covariates and each individual's ID (to account for multiple longitudinal scans per individual) and family ID (to account for the presence of dizygotic twin pairs and siblings in the sample) as nested random effects. All linear mixedeffects models discussed below were fitted using the $\mathrm{R}$ package nlme version 3.1-128 (Pinheiro et al., 2016). Sex was treated as a categorical variable coded as "M" or "F." SES and age variables were both centered. Individual ID and family ID were specified as nested random effects and were fitted with random intercepts.

The decision to present core results with SES, sex, and age as main effects was made after first ruling out the presence of extensive interactions between these variables (i.e., SES $\times$ sex or SES $\times$ age interactions) in predicting structure or vertex-level anatomical variation effect on each anatomical variable. For most structures, there was no significant interaction between fixed-effects variables; the few cases in which interactions were found will be noted in the text. Otherwise, anatomy for the $i$ th family's $j$ th individual's $k$ th time point was modeled as follows:

$$
\begin{aligned}
\text { Anatomy }_{\mathrm{ijk}}=\text { Intercept } & +\mathrm{d}_{\mathrm{i}}+\mathrm{d}_{\mathrm{ij}}+\beta_{1 \mathrm{~A}}(\mathrm{SES}-\text { mean SES }) \\
& +\beta_{2 \mathrm{~A}}(\text { age }- \text { mean age })+\beta_{3 \mathrm{~A}}(\mathrm{sex})+\mathrm{e}_{\mathrm{ijk}}[\mathrm{A}]
\end{aligned}
$$

Dependent volumetric variables of interest included total brain volume (TBV; the sum of intracranial gray and white matter volume), total intracranial gray matter volume (GMV) and total intracranial white matter volume (WMV), total bilateral volume of the cortex $(\mathrm{CV})$, total cortical surface area (SA) and mean cortical thickness (CT), and total bilateral volume of each subcortical structure (i.e., "bulk" hippocampal, amygdalar, thalamic, striatal, and pallidal volumes). To aid comparison of the associations between SES and these diverse volumetric indices, we also estimated the standardized effect for each SES-volume association by re-running model $[\mathrm{A}]$ above after centering and scaling all variables so that the resulting $\beta_{1}$ coefficient would index the SD shift in volume with one SD increase in SES (i.e., a decrease of $\sim 18$ Hollingshead points).

Vertex-level anatomical variables of interest included cortical thickness and surface area at each of 80,962 cortical vertices and SA at each of 26,401 subcortical vertices (hippocampus, 1152 left/1215 right; amygdala, 1473 left/ 1405 right; thalamus, 3016 left/3108 right; striatum, 6450 left/6178 right; pallidum, 1266 left/1138 right). Vertex-specific $\beta_{1}$ coefficients were visualized on the corresponding cortical or subcortical surface after applying a false discovery rate (FDR) correction for multiple comparisons. FDR corrections were calculated separately across the left and right cortical hemispheres and the left and right subcortical structures with $q$ (the expected proportion of false rejections of the null hypothesis) set to 0.05 .

Finally, we probed the relation among SES, anatomical metrics, and cognition in two ways. First, for all structures that showed a significant main effect of SES in model [A], we tested the robustness of this effect to the inclusion of full-scale IQ as a covariate in the linear mixed-effects models. Separate main effects of SES and IQ were included after first ruling out the presence of a significant SES by IQ interactive effect. For these analyses, anatomy for the $i$ th family's $j$ th individual's $k$ th time point was modeled as follows:

$$
\begin{aligned}
\text { Anatomy }_{\mathrm{jk}}= & \text { Intercept }+\mathrm{d}_{\mathrm{i}}+\mathrm{d}_{\mathrm{ij}}+\beta_{1 \mathrm{~B}}(\mathrm{SES}-\text { mean SES }) \\
& +\beta_{2 \mathrm{~B}}(\mathrm{IQ}-\text { mean IQ })+\beta_{3 \mathrm{~B}}(\text { age }- \text { mean age }) \\
& +\beta_{4 \mathrm{~B}}(\text { sex })+\mathrm{e}_{\mathrm{ijk}}[\mathrm{B}]
\end{aligned}
$$

Linear age terms were used in models $[\mathrm{A}]$ and $[\mathrm{B}]$ after verifying that this simple parameterization of age yielded identical SES findings to models run with age parameterized as a nonlinear spline (which could allow for nonlinear age effects). Additionally, models [A] and [B] were re-run for each anatomical metric within the subset of participants $(n=534)$ who self-endorsed the federal race category of "white" and all main effects held.

To complement our mixed-effects models and to further probe the nature of SES-anatomy associations, we directly tested the relationship between SES and intra-individual change in brain anatomy for the 344 individuals with more than one MRI scan. For each anatomical variable, 
Table 2. Associations between SES and measures of cortical and subcortical anatomy

\begin{tabular}{|c|c|c|c|c|c|c|}
\hline \multirow[b]{2}{*}{ Global anatomical Metric } & \multicolumn{2}{|c|}{$\begin{array}{l}\text { Model }[\mathrm{A}] \text { anatomy } \sim \beta_{1 \mathrm{~A}}(\mathrm{SES})+ \\
\text { age }+ \text { sex }\end{array}$} & \multicolumn{4}{|c|}{ Model $[\mathrm{B}]$ anatomy $\sim \beta_{1 \mathrm{~B}}(\mathrm{SES})+\beta_{2 \mathrm{~B}}(\mathrm{IQ})+$ age + sex } \\
\hline & $\beta_{1 \mathrm{~A}}(\mathrm{SE})$ & $p_{1 \mathrm{~A}}$ & $\beta_{1 \mathrm{~B}}(\mathrm{SE})$ & $p_{1 \mathrm{~B}}$ & $\beta_{2 \mathrm{~B}}(\mathrm{SE})$ & $p_{2 B}$ \\
\hline Total brain volume $\left(\mathrm{cm}^{3}\right)$ & $1217.0(263.0)$ & $<0.001$ & $872.3(268.3)$ & 0.001 & $1645.8(353.9)$ & $<0.001$ \\
\hline Grey matter volume $\left(\mathrm{cm}^{3}\right)$ & $773.6(168.1)$ & $<0.001$ & $539.7(171.1)$ & 0.002 & $1115.2(226.6)$ & $<0.001$ \\
\hline White matter volume $\left(\mathrm{cm}^{3}\right)$ & $444.3(117.6)$ & $<0.001$ & $331.1(121.2)$ & 0.007 & $538.1(159.8)$ & $<0.001$ \\
\hline Cortical volume $\left(\mathrm{cm}^{3}\right)$ & $642.4(135.3)$ & $<0.001$ & $458.5(137.8)$ & 0.001 & $876.7(182.6)$ & $<0.001$ \\
\hline Total surface area $\left(\mathrm{cm}^{2}\right)$ & $144.1(31.1)$ & $<0.001$ & $111.6(31.9)$ & $<0.001$ & $156.0(41.7)$ & $<0.001$ \\
\hline Mean cortical thickness $(\mathrm{cm})$ & $0.00086(0.00034)$ & 0.0127 & $0.00047(0.00035)$ & 0.186 & $0.0018(0.0005)$ & $<0.001$ \\
\hline Hippocampus volume $\left(\mathrm{cm}^{3}\right)$ & $3.56(1.08)$ & 0.001 & $2.58(1.12)$ & 0.022 & $4.59(1.54)$ & 0.003 \\
\hline Amygdala volume $\left(\mathrm{cm}^{3}\right)$ & $1.22(0.51)$ & 0.018 & $0.87(0.53)$ & 0.10 & $1.64(0.74)$ & 0.027 \\
\hline Thalamus volume $\left(\mathrm{cm}^{3}\right)$ & $9.10(2.21)$ & $<0.001$ & $6.89(2.29)$ & 0.003 & $10.44(3.11)$ & $<0.001$ \\
\hline Striatum volume $\left(\mathrm{cm}^{3}\right)$ & $7.72(3.76)$ & 0.042 & $4.78(3.89)$ & 0.22 & $13.94(5.07)$ & 0.006 \\
\hline Pallidum volume $\left(\mathrm{cm}^{3}\right)$ & $0.72(0.57)$ & 0.21 & $0.41(0.59)$ & 0.49 & $1.47(0.80)$ & 0.067 \\
\hline
\end{tabular}

Covariates in both models $[A]$ and $[B]$ include sex, centered age, and each individual's ID and family ID.

we calculated an annual rate of change for each individual. Anatomical change was then modeled using a linear model with main effects of SES, mean scan age, and sex and the interaction between SES and mean scan age. The association between annual rate of change and SES was investigated for each of the anatomical variables considered in the mixed models above: TBV, GMV, WMV, CV, total SA, mean CT, five subcortical volumes, and vertexwise CT, cortical SA, and subcortical SA.

Finally, we conducted mediation analyses to investigate the extent to which the global and vertexwise structural associations with SES mediate the association between SES and IQ. For the mediation tests with a single global mediator, we used the Mediation package in $\mathrm{R}$ (Tingley et al., 2014), with SES as the independent variable (treatment) and full-scale IQ as the dependent variable. The mediate() function estimates the average causal mediation effect (ACME) and the average direct effect (ADE), which together sum to the total effect of the treatment (i.e., SES) on the outcome (i.e., IQ). The proportion mediated, which we report, represents the size of the ACME relative to the total effect. Within the map of cortical vertices that showed a significant association with SES, we tested the mediating role of each vertex using the MultiMed package in $\mathrm{R}$ (Boca et al., 2014), which implements a permutation approach with joint correction to test multiple mediators simultaneously.

Interpretation of anatomical results. To systematically investigate the functional implications of SES effects, we submitted the MNI coordinates of peak SES effects on surface area and cortical thickness to Neurosynth (Yarkoni et al., 2011), an online platform that extracts and synthesizes brain activation patterns and psychological terms across $>11,000$ functional neuroimaging publications. It can be used to generate correlations between the meta-analytic coactivation map for a given point of interest in the brain and each of the terms in the Neurosynth database. Using Neurosynth, we identified cognitive and psychological terms that frequently cooccur in the literature with functional activations similar to the observed pattern of SES effects on cortical morphology.

\section{Results}

\section{Participant characteristics}

Participant characteristics are detailed in Table 1. Hollingshead SES in our sample ranged from a high of 20 to a low of 95 after three scans from individuals with exceptionally low SES scores (Hollingshead SES $=115, \mid z$-score $\mid>4$ ) were removed. The complete Hollingshead scale ranges from 20 to 134 and approximately one-quarter of our 623 participants received the highest SES score of 20 (indicating that one or both of their parents received a graduate professional degree and employment in the highest occupation category). Therefore, our sample's distribution of Hollingshead scale scores does not capture the most severely socioeconomically disadvantaged children and it is relatively enriched for children with highly educated and highly employed parents. Throughout the text below, SES is referred to using conventional directionality such that "higher SES" refers to a lower Hollingshead score, and reported positive associations with SES are thus negatively correlated with Hollingshead score. Within the cross-sectional sample of 623 individuals, SES and IQ were significantly, positively correlated, $r_{(621)}=0.31, p<0.001$.

\section{SES and measures of brain volume}

We found a strong positive association between SES and total brain volume $\left(\beta_{1 \mathrm{~A}}=1217.0, p<0.001\right)$. A positive association with SES was also seen for total white and gray matter volumes, as well as for total volume of the cortical sheet (Table 2). Separate examination of the two determinants of cortical volume identified a strong positive association between SES and total cortical surface area $\left(\beta_{1 \mathrm{~A}}=144.1, p<0.001\right)$ and a weaker positive association between SES and mean cortical thickness $\left(\beta_{1 \mathrm{~A}}=\right.$ $0.00086, p=0.01)$. Greater SES was also significantly associated with greater bilateral volume of all subcortical volumes examined except the pallidum (Table 2). Analysis of standardized effect sizes for SES associations with regional brain volumes revealed that SES was most strongly related to total brain volume and total surface area such that an SD increase in SES was associated with a $0.17 \mathrm{SD}$ increase in each of these metrics (Fig. 1A). Among subcortical structures, SES had the greatest effect on thalamic volume such that a SD increase in SES was associated with a 0.15 SD increase in thalamic volume (Fig. $1 A$ ).

These above associations between SES and brain anatomy were all stable between ages 5 and 25 and comparable between males and females, with two exceptions: (1) the association between SES and total cortical surface area was modified by sex such that there was a stronger SES effect on surface area for males than females $\left(\right.$ SES $\times$ sex interaction effect: $\beta_{\text {interaction }}=122.2, p=$ 0.019; SES main effects: $\beta_{1 \mathrm{~A}, \mathrm{MALES}}=198.7, p<0.001 /$ $\beta_{1 \mathrm{~A}, \mathrm{FEMALES}}=77.6, p=0.066$ ) and (2) the association between SES and hippocampal volume was modified by age such that the effect of SES on hippocampal volume grows with age (SES $\times$ age interaction effect: $\beta_{\text {interaction }}=0.19, p=0.003$ ).

\section{SES and cortical morphology}

After establishing that SES was associated with total surface area and mean cortical thickness (Table 2), we tested for regional specificity of these associations through vertex-level analysis of SES associations at 80,962 points (vertices) across the cortical sheet. Vertex-level analyses established that the robust association between greater SES and greater total cortical SA was underpinned by statistically significant positive associations between SES and regional SA within a distributed set of largely bilateral 
A

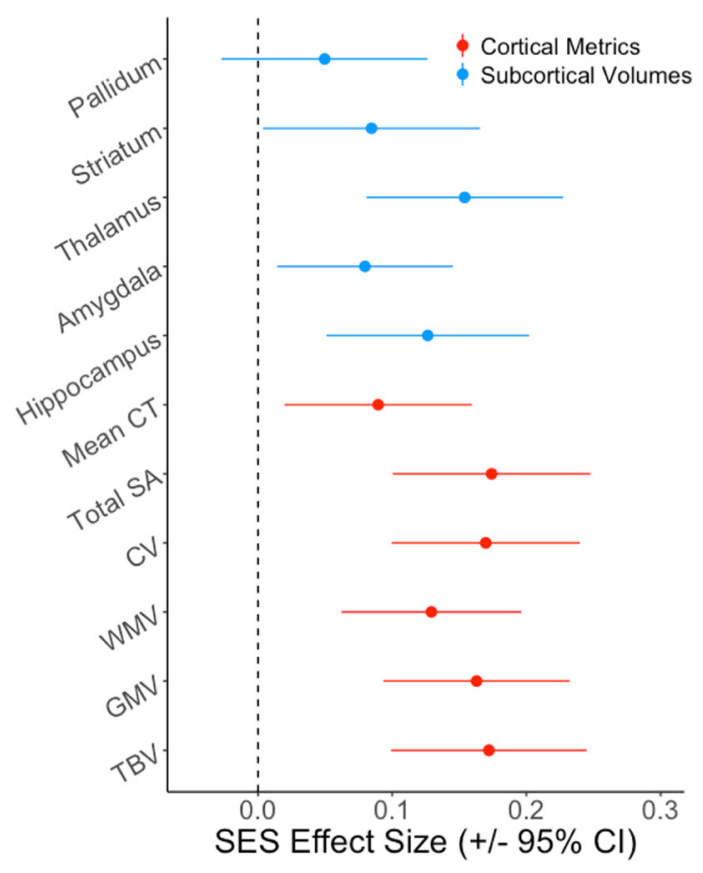

B
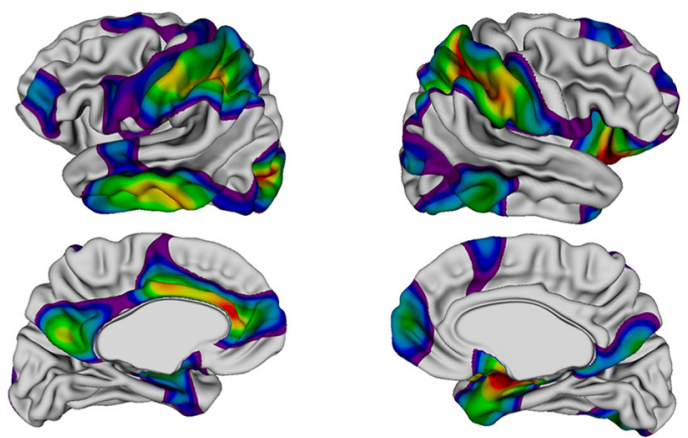

2.3

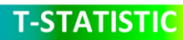

4.7

C

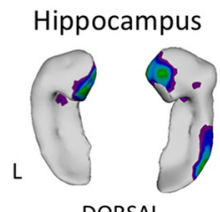

Amygdala

Thalamus

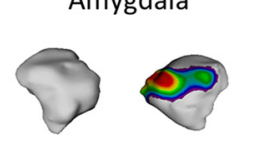

DORSAL
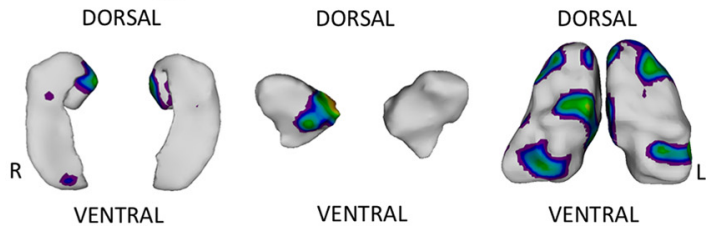

Figure 1. Main effects of SES on global and local anatomy after controlling for age and sex. $A$, Standardized effect size of SES on each global cortical and subcortical brain measure estimated using scaled variables: TBV, GMV, WMV, CV, SA, mean CT, hippocampus volume, amygdala volume, thalamus volume, striatum volume, and pallidum volume. $B$, Cortical surface regions that show a significant positive association of surface area with childhood SES. C, Subcortical surface regions that show a significant positive association of surface area with childhood SES.

cortical areas including the lateral prefrontal, anterior cingulate, and lateral temporal and superior parietal lobule regions (Fig. 1B). Associations between SES and vertex-level SA were not significantly modified by age or sex after correction for multiple comparisons.

Associations between SES and regional cortical thickness were much more localized than observed for regional SA. Specifically, we identified a single locus of significant positive association between SES and CT in the right supramarginal gyrus.

\section{SES and subcortical morphology}

We investigated the spatial specificity of observed associations between SES and bilateral subcortical volumes by modeling surface area at each vertex across the surface of the hippocampus, amygdala, thalamus, and striatum (Fig. 1C). Hippocampal effects were concentrated bilaterally on the medial surface and neighboring medial surfaces of the right amygdala also showed a significant positive association with SES. The association between SES and thalamic surface area was localized primarily to the ventral posterior and ventral lateral thalamus. No other subcortical structures showed statistically significant shape associations with SES after correcting for multiple comparisons across vertices.

\section{SES and intra-individual anatomical change}

To complement our mixed-model analyses and directly model intra-individual change, we further investigated the relationships between SES and annual rate of anatomical change of each of the cortical and subcortical metrics discussed above. Among the 344 individuals with more than one MRI scan, there was no significant relationship between SES and change in any of the global anatomical metrics (TTV, GMV, WMV, CV, total SA, mean CT, or any subcortical volume). These results are fully consistent with the previously noted lack of significant SES $\times$ age interactive effect on any global anatomical metric, with the sole exception of findings for hippocampal volume. Results from analyses of intra-individual change and from mixed-model analysis were also largely consistent for vertex-level measures of cortical surface area and thickness: both analytic approaches suggested that age does not modulate SES effects on thickness or surface area across most of the cortical sheet or on area across subcortical surfaces. We observed two spatially limited exceptions to this convergence between analytic techniques in that analysis of intra-individual change uniquely identified a statistically significant negative relationships between SES and cortical thickness change in the left middle temporal gyrus and the left superior parietal lobule after FDR correction and a statistically significant, negative relationship between SES and surface area change in a single locus on the left dorsal thalamus. A figure showing results of the vertexwise change analyses is available on figshare (McDermott, 2018).

\section{Separating main effects of SES and IQ on anatomy}

Because of the noted strong association between SES and IQ (Fig. $2 A$ ), we next re-ran linear mixed-effects models with full-scale IQ as a covariate to parse main effects of SES and cognition on neuroanatomy. We observed positive associations between IQ and all global measures of cortical and subcortical anatomy (Table 2). The main effect of SES on each global metric was reduced in magnitude by the addition of IQ to the model; for mean cortical thickness, bulk amygdalar volume, and bulk striatal volume, the main effect of SES was no longer significant (Table 2), although SES continued to show a significant positive association with TBV, GMV, WMV, CV, total cortical SA, and bilateral hippocampal and thalamic volumes after controlling for IQ. There were no significant SES $\times$ IQ interactive effects on any global metrics.

We next probed the spatial patterning of IQ versus SES main effects on cortical surface area. After FDR correction for multiple 


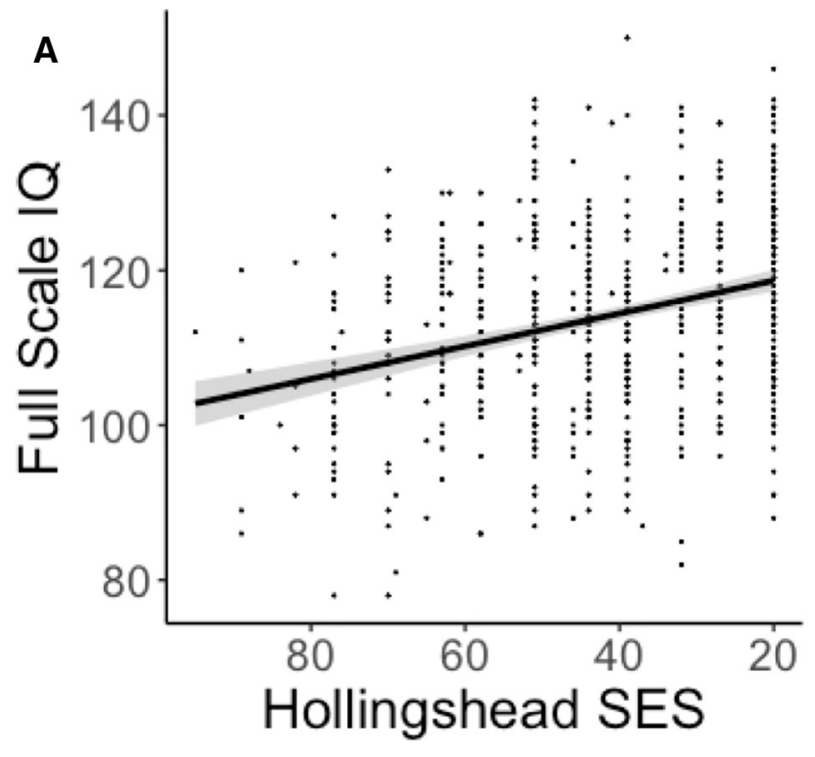

B $\mathrm{SA} \sim B_{1}(\mathrm{SES})+B_{2}(\mathrm{IQ})+$ age + sex
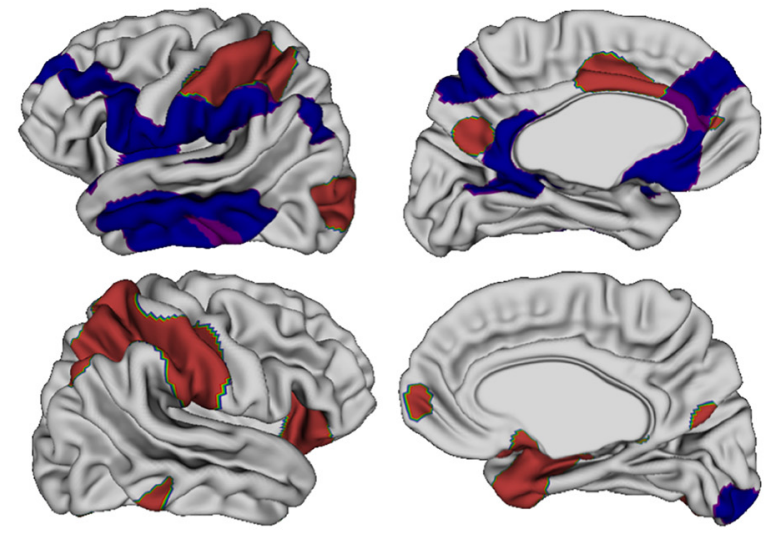

C

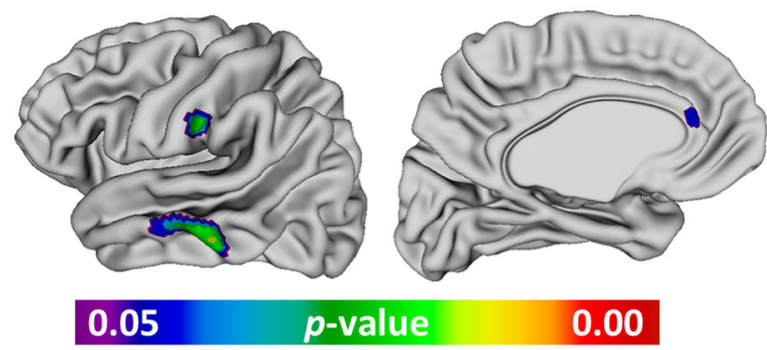

Figure 2. $\quad \boldsymbol{A}$, Correlation between childhood SES and full-scale IQ in a cross-sectional sample of 623 individuals, $r_{(621)}=0.31, p<0.001$. B, Map of the main effects of SES and IQ on cortical surface area. Significant SES main effect is represented in red, IQ main effect in blue, and overlapping main effects in purple. Together, these main effects resemble the map of main SES effects on cortical SA without IQ as a covariate (Fig. 1B). C, Mediation analysis including surface area at each vertex as a separate mediator revealing regions of the left hemisphere that significantly mediate the relationship between SES and IQ.

comparisons, childhood SES and IQ were both positively associated with regional cortical SA (Fig. 2B). Specifically, when including IQ as a covariate, the main effect of SES was restricted to the bilateral superior parietal, right orbitofrontal, left inferior temporal, and bilateral medial prefrontal cortices. The main effect of IQ, in contrast, was localized to the left inferior and middle temporal, left inferior parietal, and left medial frontal regions. Strikingly, the regional maps of separate SES and IQ effects on cortical surface area together (Fig. $2 B$ ) resemble the regional effects of SES when IQ is not included as a covariate (Fig. $1 B$ ). In other words, the main effect of SES on cortical surface area appears to be separable into effects related to the strong IQ differences across SES localized to the left-lateralized perisylvian and medial prefrontal cortices and SES-SA associations that are independent from associated variation in general cognitive ability localized to bilateral parietal and frontal cortices.

\section{Structural measures mediate the association between SES and IQ}

In addition to testing the effects of IQ as a covariate in SES-brain associations, we also probed the relation among SES, cognition, and anatomy by investigating whether any of the structural brain measures found to be significantly associated with SES might mediate the association between SES and IQ in our crosssectional dataset of 623 individuals. Using the Mediate package in $\mathrm{R}$ (Tingley et al., 2014), we estimated the proportion of the total relation between SES and IQ that was accounted for by each anatomical mediator. Among global cortical measures, total brain volume (proportion mediated $=0.148, p<0.001$ ), gray matter volume (proportion mediated $=0.145, p<0.001$ ), white matter volume (proportion mediated $=0.077, p<0.001$ ), cortical volume (proportion mediated $=0.146, p<0.001$ ), total surface area (proportion mediated $=0.114, p<0.001$ ), and mean cortical thickness (proportion mediated $=0.049, p=0.01$ ) each partially mediated the relation between SES and IQ. Subcortical volumes also partially mediated the association (hippocampus, proportion mediated $=0.052, p<0.001$; amygdala, proportion mediated $=0.034 ; p=0.02$; thalamus, proportion mediated $=0.087 ; p<0.001 ;$ striatum, proportion mediated $=$ $0.033, p=0.01$ ).

Finally, we extended the mediation analysis to investigate whether there was a regional specificity to the partial mediation of SES and IQ by cortical and subcortical surface area. To do so, we submitted all of the vertices in each hemisphere that showed a significant association with SES to a multiple mediation analysis using the MultiMed package in R (Boca et al., 2014). Three cortical regions, all in the left hemisphere, showed significant mediation of the SES association with IQ: the middle temporal gyrus, supramarginal gyrus, and anterior cingulate cortex (Fig. 2C). Notably, these largely overlapped with the cortical vertices that showed shared main effects of SES and IQ on surface area (regions in purple, Fig. 2B).

\section{Functional implications of anatomical results}

Coordinates of peak regional SES associations with cortical surface area and cortical thickness were submitted to Neurosynth (Yarkoni et al., 2011), a meta-analytic repository of structurefunction relationships. The top five cognitive and psychological terms with the highest meta-analytic coactivation correlation coefficient $r$ are displayed in Table 3 after redundant terms and anatomical terms were removed. This platform provided evidence that the spatial effects of SES are localized to regions preferentially associated with sensorimotor functions, as well as language, memory, and emotional processing, which have each been shown to exhibit SES differences (Farah et al., 2006; Noble et al., 2007; Kim P et al., 2013).

\section{Discussion}

Here, with a large, longitudinal, single-site neuroimaging sample, we both replicate and extend findings on the relation between childhood SES and structural brain anatomy in a number of key directions. 
Table 3. Locations of peak SES associations with cortical morphology and top functional associations as indicated by Neurosynth

\begin{tabular}{|c|c|c|c|c|c|}
\hline \multirow[b]{2}{*}{ Region of peak vertex } & \multirow[b]{2}{*}{$t$} & \multicolumn{3}{|c|}{ MNI coordinates } & \multirow[b]{2}{*}{ Top Neurosynth terms } \\
\hline & & $x$ & $y$ & $z$ & \\
\hline \multicolumn{6}{|l|}{ Surface area } \\
\hline R orbitofrontal cortex & 4.6 & 20 & 19 & -22 & Emotion; reward; affective; fear; regulation \\
\hline L occipital pole & 4.6 & -35 & -85 & 7 & Visual; objects; motion; orthographic; reading \\
\hline L inferior parietal cortex & 4.6 & -44 & -38 & 49 & Execution; motor; movement; working mem.; spatial \\
\hline L anterior cingulate gyrus & 4.4 & -4 & 36 & 10 & Default mode (DM); reward; self-referential; emotional; autobiographical \\
\hline R middle temporal gyrus & 3.5 & 61 & -42 & -10 & Semantic; language; comprehension; sentence; word \\
\hline R precuneus & 3.4 & 4 & -68 & 28 & DM; autobiographical mem.; mem. retrieval; self-referential; episodic mem. \\
\hline R superior frontal gyrus & 3.4 & 5 & 62 & -5 & DM; autobiographical mem.; self-referential; social; theory of mind \\
\hline \multicolumn{6}{|r|}{ 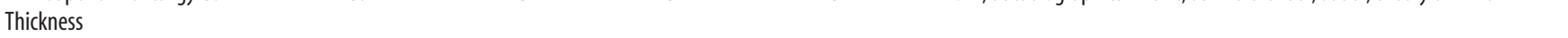 } \\
\hline R supramarginal gyrus & 4.3 & 59 & -19 & 33 & Somatosensory; motor; tactile; movement; pain \\
\hline
\end{tabular}

First, consistent with previously replicated findings, we find that SES is positively associated with global brain measures including gray matter volume (Hanson et al., 2013; Luby et al., 2013; Mackey et al., 2015; Gianaros et al., 2017), cortical surface area (Noble et al., 2015; Gianaros et al., 2017), cortical thickness (Mackey et al., 2015; Noble et al., 2015), and hippocampal volume (Noble et al., 2012, 2015; Luby et al., 2013; Hair et al., 2015). Although previous findings regarding white matter volume and amygdala volume have been mixed (Jednoróg et al., 2012; Luby et al., 2013; Noble et al., 2015; Gianaros et al., 2017), we identify significant positive SES associations with both WMV and amygdala volume. We extend analyses of SES associations with subcortical anatomy to provide the first evidence that greater childhood SES is associated with larger bilateral volumes of the thalamus and striatum. The thalamic finding is especially notable given reported associations between thalamic volume and cognitive performance (Van Der Werf et al., 2001; Xie et al., 2012). Comparative analyses of SES associations using standardized effect sizes ranks total cortical SA and bilateral thalamic volume as the morphometric properties of the cortex and subcortex, respectively, which show the largest effect-size relations with SES.

It is of particular note that, with the exception of hippocampal volume, we found the above noted SES associations with global cortical and subcortical anatomical metrics to be fixed between ages 5 and 25. This conclusion was supported by two complementary analytic approaches: mixed-model testing for significant SES $\times$ age interactive effects and direct analysis of SES effects on intra-individual rates of anatomical change. Other crosssectional studies with age ranges similar to the population examined here have likewise reported limited or no SES $\times$ age interactions (Lawson et al., 2013; Noble et al., 2015); however, a longitudinal study of children under the age of 4 found that SES disparities in GMV grow with age (Hanson et al., 2013). These findings suggest that SES and age may interact differently across developmental time, with developmentally dynamic associations early in life, which subsequently stabilize. Future longitudinal studies bridging the gap between infancy and adolescence may help to clarify the temporal dynamics of SES associations with brain development.

Second, our study advances understanding of the neuroanatomical correlates of SES by using surface-based algorithms to parse associations between SES and anatomy of the cortical sheet. We observe SES associations with cortical surface area in a number of largely bilateral regions including lateral prefrontal, anterior cingulate, lateral temporal, and superior parietal lobule regions. These SES-surface area associations largely correspond to the map of associations between parental education and cortical surface area presented by Noble et al. (2015). Functional interpretation with Neurosynth (Yarkoni et al., 2011) suggests that the cortical regions that we found to show morphological associations with SES are preferentially involved in networks that underlie sensorimotor functions, as well as language, memory, and emotional regulation.

Third, we provide the first systematic examination of SES associations with subcortical shape, which indicates that variations in childhood SES are associated with focal differences in hippocampal, amygdalar, and thalamic anatomy (Fig. 1C). Histological and functional connectivity neuroimaging studies suggest that the ventral lateral and ventral posterior thalamic nuclei are preferentially connected to frontoparietal cortical systems that subserve primarily sensorimotor functions (Jones, 1985; Kim D-J et al., 2013). Strikingly, we also detect strong associations between SES and surface area within these cortical targets of ventral thalamic nuclei, suggesting that anatomical correlations of childhood SES variation may be organized by the topography of corticosubcortical connectivity.

Finally, we demonstrate convergent anatomical correlates of the strong relation between SES and IQ using two independent methods. Modeling the main effects of both IQ and SES on vertexwise surface area reveals that the map of statistical SES effects can be fractionated into distinct SES and IQ effects. Additionally, we demonstrate that many of the anatomical correlates of SES significantly mediate the relation between SES and IQ. We expand upon previous results that found significant SES-cognition mediation by whole-brain surface area (Noble et al., 2015) by conducting cross-sectional mediation tests at each cortical vertex that had a significant SES-surface area association. Specifically, we find that vertices in the left middle temporal gyrus, supramarginal gyrus, and anterior cingulate, the same three regions that showed a main effect of IQ when controlling for SES, mediate the relation between SES and IQ. These three regions are known to be involved in language (Vannest et al., 2009) and cognitive control (Shackman et al., 2011).

A mediation analysis allows us to test one possible pathway between SES, brain anatomy, and cognition and we show that SES may exert some of its effect on cognition by altering structural brain development, particularly in regions associated with language and learning. However, it is important to note that this pathway represents only one possible set of interactions between childhood environment, anatomy, and cognition. Farah (2017) provides a succinct review of the main processes that may operate to exacerbate neural and psychological SES disparities: the social 
causation hypothesis suggests that the environmental conditions associated with different levels of SES influence brain structure and function, whereas the social selection hypothesis suggests that genetic factors in parents that both affect their cognition and predispose them to a certain SES are then transmitted to their children. These processes likely both operate in concert and interact to some degree. Because of the inherent observational nature of studies of SES, it is difficult to determine to what extent the anatomical correlates of SES that we report here may reflect the shared genetic effects on SES and brain development versus direct effects of SES on brain development. Nevertheless, our findings help to pinpoint cortical and subcortical systems that represent candidate biological substrates for these diverse causal pathways.

\section{Caveats and future directions}

One important limitation of this sample is the composite nature of the Hollingshead two-factor index of SES. The field has lately recognized the importance of differentiating between the effects of separate components of socioeconomic status (Duncan and Magnuson, 2012). A few studies have already begun to identify differential effects of parental education and family income on structural brain development (Lawson et al., 2013; Noble et al., 2015). However, when data collection for this sample began in the 1990s, the Hollingshead was selected for its widespread use and ease of measurement and only the single derived SES score was recorded initially. As future studies are designed, it will be important to report on more nuanced factors that compose SES and may have unique effects on brain development and thus serve as specific targets for intervention.

It is also important to note that the subjects in this sample are not representative of the socioeconomic distribution in the United States. Sample composition is known to affect conclusions about the normative trajectory of brain development (LeWinn et al., 2017). Additionally, some studies have suggested that socioeconomic variables relate most strongly to brain development among the most disadvantaged children (Noble et al., 2015) and the fact that we did not detect such a gradient may be due to our unrepresentative SES distribution. The average IQ of our subjects was also higher than the expected mean of 100 (Table 1); however, IQ is known to increase across generations in the general population, so the high mean IQ may be partially attributable to the use of the same IQ assessment across the multiple decades of this study (Flynn, 1987). Finally, most investigations of SES and brain development to date, including this study, have sampled from Western nations in general and the United States in particular. Further research is necessary to explore how SES associations with brain development play out across other nations and cultures. Although we cannot generalize across the socioeconomic, cognitive, or cultural spectrums, it is notable that we found detectible SES-linked differences in cognitive ability and structural brain development within a typically developing cohort lacking frank socioeconomic deprivation.

\section{Conclusion}

Childhood socioeconomic status is a complex construct that influences the physical and psychosocial environment in which a child develops. Here, we demonstrate regionally specific associations between childhood SES and both cortical and subcortical morphology. Our findings inform ongoing efforts to clarify the spatiotemporal patterning of SES-related neuroanatomical variation and its relation to cognitive outcomes such as IQ. Definition of these neuroanatomical associations may ultimately provide candidate biological substrates against which to test potential mechanis- tic pathways between SES and cognitive and health outcomes. The results presented here do not establish a direct causal pathway between SES and brain development, nor do they indicate that childhood SES exerts a deterministic effect on development. Rather, by resolving neuroanatomical substrates that vary closely with SES, we contribute new biological information to a growing field of multidisciplinary research that ultimately aims to reduce SES variation in health and achievement.

\section{References}

Ad-Dab'bagh Y, Einarson D, Lyttelton O, Muehlboeck J-S, Mok K, Ivanov O, Vincent R, Lepage C, Lerch J, Fombonne E, Evans AC (2006) The CIVET image-processing environment: A fully automated comprehensive pipeline for anatomical neuroimaging research. Paper presented at the 12th Annual Meeting of the Organization for Human Brain Mapping, Florence, Italy, June.

Boca SM, Sinha R, Cross AJ, Moore SC, Sampson JN (2014) Testing multiple biological mediators simultaneously. Bioinformatics 30:214-220. CrossRef Medline

Brito NH, Noble KG (2014) Socioeconomic status and structural brain development. Front Neurosci 8:276. CrossRef Medline

Brooks-Gunn J, Duncan GJ (1997) The effects of poverty on children. Future Child 7:55-71. CrossRef Medline

Chakravarty MM, Bertrand G, Hodge CP, Sadikot AF, Collins DL (2006) The creation of a brain atlas for image guided neurosurgery using serial histological data. Neuroimage 30:359-376. CrossRef Medline

Chakravarty MM, Steadman P, van Eede MC, Calcott RD, Gu V, Shaw P, Raznahan A, Collins DL, Lerch JP (2013) Performing label-fusionbased segmentation using multiple automatically generated templates. Hum Brain Mapp 34:2635-2654. CrossRef Medline

Cocosco CA, Zijdenbos AP, Evans AC (2003) A fully automatic and robust brain MRI tissue classification method. Med Image Anal 7:513-527. CrossRef Medline

Collins DL, Neelin P, Peters TM, Evans AC (1994) Automatic 3D intersubject registration of $\mathrm{MR}$ volumetric data in standardized talairach space. J Comput Assist Tomogr 18:192-205. CrossRef Medline

Draganski B, Kherif F, Klöppel S, Cook PA, Alexander DC, Parker GJM, Deichmann R, Ashburner J, Frackowiak RSJ (2008) Evidence for segregated and integrative connectivity patterns in the human basal ganglia. J Neurosci 28:7143-7152. CrossRef Medline

Duncan GJ, Magnuson K (2012) Socioeconomic status and cognitive functioning: moving from correlation to causation. Wiley Interdiscip Rev Cogn Sci 3:377-386. CrossRef Medline

Farah MJ (2017) The neuroscience of socioeconomic status: correlates, causes, and consequences. Neuron 96:56-71. CrossRef Medline

Farah MJ, Shera DM, Savage JH, Betancourt L, Giannetta JM, Brodsky NL, Malmud EK, Hurt H (2006) Childhood poverty: specific associations with neurocognitive development. Brain Res 1110:166-174. CrossRef Medline

Flynn, JR (1987) Massive IQ gains in 14 nations: What IQ tests really measure. Psychological Bulletin. 101:171-191. CrossRef

Gianaros PJ, Kuan DC-H, Marsland AL, Sheu LK, Hackman DA, Miller KG, Manuck SB (2017) Community socioeconomic disadvantage in midlife relates to cortical morphology via neuroendocrine and cardiometabolic pathways. Cereb Cortex 27:460-473. Medline

Giedd JN, Blumenthal J, Jeffries NO, Castellanos FX, Liu H, Zijdenbos A, Paus T, Evans AC, Rapoport JL (1999) Brain development during childhood and adolescence: a longitudinal MRI study. Nat Neurosci 2:861-863. CrossRef Medline

Hair NL, Hanson JL, Wolfe BL, Pollak SD (2015) Association of child poverty, brain development, and academic achievement. JAMA Pediatr 169: 822-829. CrossRef Medline

Hanson JL, Hair N, Shen DG, Shi F, Gilmore JH, Wolfe BL, Pollak SD (2013) Family poverty affects the rate of human infant brain growth. PLoS One 8:e80954. CrossRef Medline

Hill WD, Hagenaars SP, Marioni RE, Harris SE, Liewald DCM, Davies G, Okbay A, McIntosh AM, Gale CR, Deary IJ (2016) Molecular genetic contributions to social deprivation and household income in UK biobank. Curr Biol 26:3083-3089. CrossRef Medline

Hollingshead AB (1957) Two factor index of social position. New Haven: Yale University Press.

Holz NE, Boecker R, Hohm E, Zohsel K, Buchmann AF, Blomeyer D, Jennen- 
Steinmetz C, Baumeister S, Hohmann S, Wolf I, Plichta MM, Esser G, Schmidt M, Meyer-Lindenberg A, Banaschewski T, Brandeis D, Laucht M (2015) The long-term impact of early life poverty on orbitofrontal cortex volume in adulthood: results from a prospective study over 25 years. Neuropsychopharmacology 40:996-1004. CrossRef Medline

Jednoróg K, Altarelli I, Monzalvo K, Fluss J, Dubois J, Billard C, DehaeneLambertz G, Ramus F (2012) The Influence of Socioeconomic Status on Children's Brain Structure. PLoS ONE 7:e42486. CrossRef Medline

Jones EG (1985) The Thalamus. New York: Plenum.

Kendler KS, Turkheimer E, Ohlsson H, Sundquist J, Sundquist K (2015) Family environment and the malleability of cognitive ability: a swedish national home-reared adopted-away cosibling control study. Proc Natl Acad Sci U S A 112:4612-4617. CrossRef Medline

Kim D-J, Park B, Park H-J (2013) Functional connectivity-based identification of subdivisions of the basal ganglia and thalamus using multilevel independent component analysis of resting state fMRI. Hum Brain Mapp 34:1371-1385. CrossRef Medline

Kim P, Evans GW, Angstadt M, Ho SS, Sripada CS, Swain JE, Liberzon I, Phan KL (2013) Effects of childhood poverty and chronic stress on emotion regulatory brain function in adulthood. Proc Natl Acad Sci U S A 110: 18442-18447. CrossRef Medline

Lawson GM, Duda JT, Avants BB, Wu J, Farah MJ (2013) Associations between children's socioeconomic status and prefrontal cortical thickness. Dev Sci 16:641-652. CrossRef Medline

Lerch JP, Carroll JB, Spring S, Bertram LN, Schwab C, Hayden MR, Henkelman RM (2008) Automated deformation analysis in the YAC128 huntington disease mouse model. Neuroimage 39:32-39. CrossRef Medline

LeWinn KZ, Sheridan MA, Keyes KM, Hamilton A, McLaughlin KA (2017) Sample composition alters associations between age and brain structure. Nat Commun 8:874. CrossRef Medline

Luby J, Belden A, Botteron K, Marrus N, Harms MP, Babb C, Nishino T, Barch D (2013) The effects of poverty on childhood brain development: the mediating effect of caregiving and stressful life events. JAMA Pediatr 167:1135-1142. CrossRef Medline

MacDonald D, Kabani N, Avis D, Evans AC (2000) Automated 3-D extraction of inner and outer surfaces of cerebral cortex from MRI. Neuroimage 12:340-356. CrossRef Medline

Mackey AP, Finn AS, Leonard JA, Jacoby Senghor DS, West MR, Gabrielli CFO, Gabrieli JDE (2015) Neuroanatomical correlates of the income achievement gap. Psychol Sci 26:925-933. CrossRef Medline

McDermott C (2018) Vertexwise neuroanatomical change associations with childhood socioeconomic status. figshare. Available at https://doi. org/10.6084/m9.figshare.7089185.v2

McLoyd VC (1998) Socioeconomic disadvantage and child development. Am Psychol 53:185-204. CrossRef Medline

Noble KG, Houston SM, Brito NH, Bartsch H, Kan E, Kuperman JM, Akshoomoff N, Amaral DG, Bloss CS, Libiger O, Schork NJ, Murray SS, Casey BJ, Chang L, Ernst TM, Frazier JA, Gruen JR, Kennedy DN, Van Zijl P, Mostofsky S, et al. (2015) Family income, parental education and brain structure in children and adolescents. Nat Neurosci 18:773-778. CrossRef Medline

Noble KG, McCandliss BD, Farah MJ (2007) Socioeconomic gradients predict individual differences in neurocognitive abilities. Dev Sci 10:464480. CrossRef Medline

Noble KG, Houston SM, Kan E, Sowell ER (2012) Neural correlates of socioeconomic status in the developing human brain. Dev Sci 15:516-527. CrossRef Medline

Pinheiro J, Bates D, DebRoy S, Sarkar D, R Core Team (2016) nlme: Linear and nonlinear mixed effects models. R Package Version 31-128.

Raznahan A, Shaw P, Lalonde F, Stockman M, Wallace GL, Greenstein D, Clasen L, Gogtay N, Giedd JN (2011) How does your cortex grow? J Neurosci 31:7174-7177. CrossRef Medline
Raznahan A, Lenroot R, Thurm A, Gozzi M, Hanley A, Spence SJ, Swedo SE, Giedd JN (2012) Mapping cortical anatomy in preschool aged children with autism using surface-based morphometry. Neuroimage Clin 2:111119. CrossRef Medline

Raznahan A, Shaw PW, Lerch JP, Clasen LS, Greenstein D, Berman R, Pipitone J, Chakravarty MM, Giedd JN (2014) Longitudinal fourdimensional mapping of subcortical anatomy in human development. Proc Natl Acad Sci U S A 111:1592-1597. CrossRef

Redgrave P, Rodriguez M, Smith Y, Rodriguez-Oroz MC, Lehericy S, Bergman H, Agid Y, DeLong MR, Obeso JA (2010) Goal-directed and habitual control in the basal ganglia: implications for Parkinson's disease. Nat Rev Neurosci 11:760-772. CrossRef Medline

Reiss F (2013) Socioeconomic inequalities and mental health problems in children and adolescents: a systematic review. Soc Sci Med 90:24-31. CrossRef Medline

Ritsher JE, Warner V, Johnson JG, Dohrenwend BP (2001) Inter-generational longitudinal study of social class and depression: a test of social causation and social selection models. Br J Psychiatry Suppl 40:s84-90. Medline

Shackman AJ, Salomons TV, Slagter HA, Fox AS, Winter JJ, Davidson RJ (2011) The integration of negative affect, pain and cognitive control in the cingulate cortex. Nat Rev Neurosci 12:154-167. CrossRef Medline

Sirin SR (2005) Socioeconomic status and academic achievement: a metaanalytic review of research. Rev Educ Res 75:417-453. CrossRef

Sled JG, Zijdenbos AP, Evans AC (1998) A nonparametric method for automatic correction of intensity nonuniformity in MRI data. IEEE Trans Med Imaging 17:87-97. CrossRef Medline

Tiikkaja S, Sandin S, Hultman CM, Modin B, Malki N, Sparén P (2016) Psychiatric disorder and work life: a longitudinal study of intra-generational social mobility. Int J Soc Psychiatry 62:156-166. CrossRef Medline

Tingley D, Yamamoto T, Hirose K, Keele L, Imai K (2014) mediation: $\mathrm{r}$ package for causal mediation analysis. J Stat Softw 59:1-38. CrossRef

Trzaskowski M, Harlaar N, Arden R, Krapohl E, Rimfeld K, McMillan A, Dale PS, Plomin R (2014) Genetic influence on family socioeconomic status and children's intelligence. Intelligence 42:83-88. CrossRef Medline

Van Der Werf YD, Tisserand DJ, Visser PJ, Hofman PA, Vuurman E, Uylings HB, Jolles J (2001) Thalamic volume predicts performance on tests of cognitive speed and decreases in healthy aging: a magnetic resonance imaging-based volumetric analysis. Brain Res Cogn Brain Res 11:377385. CrossRef Medline

Vannest J, Karunanayaka PR, Schmithorst VJ, Szafkarski JP, Holland SK (2009) Language networks in children: evidence from functional MRI studies. Am J Roentgenol 192:1190-1196. CrossRef

Walhovd KB, et al (2016) Neurodevelopmental origins of lifespan changes in brain and cognition. Proc Natl Acad Sci U S A 113:9357-9362. CrossRef Medline

Watt NF (1976) Two-factor index of social position: Amherst modification. Unpublished manuscript: University of Massachusetts at Amherst.

Wechsler D (1999) Wechsler abbreviated scale of intelligence. San Antonio: The Psychological Corporation.

Wood SN (2011) Fast stable restricted maximum likelihood and marginal likelihood estimation of semiparametric generalized linear models. J R Stat Soc Ser B Stat Methodol 73:3-36. CrossRef

Xie Y, Chen YA, De Bellis MD (2012) The relationship of age, gender, and IQ with the brainstem and thalamus in healthy children and adolescents: a magnetic resonance imaging volumetric study. J Child Neurol 27:325331. CrossRef Medline

Yarkoni T, Poldrack RA, Nichols TE, Van Essen DC, Wager TD (2011) Large-scale automated synthesis of human functional neuroimaging data. Nat Methods 8:665-670. CrossRef Medline

Zijdenbos AP, Forghani R, Evans AC (2002) Automatic "pipeline" analysis of 3-D MRI data for clinical trials: application to multiple sclerosis. IEEE Trans Med Imaging 21:1280-1291. CrossRef Medline 